\title{
Diversified transcription initiation complexes expand promoter selectivity and tissue-specific gene expression
}

\author{
Andreas Hochheimer and Robert Tjian ${ }^{1}$ \\ Department of Molecular and Cell Biology, Howard Hughes Medical Institute, University of California, \\ Berkeley, California 94720-3204, USA
}

Transcription initiation is a key regulatory step in the control of gene expression. Formation of a preinitiation complex at the right time and at the right promoter is a prerequisite to executing the correct programs of mRNA synthesis. This involves the interplay of many transcription factors and a combinatorial array of cis-regulatory DNA elements. Recent findings suggest that metazoan organisms have evolved specialized transcription initiation complexes and promoter-selectivity modules that direct coordinated regulation of functionally related gene networks. Here, we summarize corroborating evidence for a highly diversified core transcription machinery directing cell type-specific and gene-selective transcription.

In multicellular organisms elaborate mechanisms have evolved to control the spatial and temporal patterns of transcription during growth, differentiation, and development. Transcriptional activation, one of the fundamental means of regulating gene expression in eukaryotes, is governed by an interconnected ensemble of multisubunit transcription factor complexes (Fig. 1; Lemon and Tjian 2000; Narlikar et al. 2002; Orphanides and Reinberg 2002). To assure the proper assembly of the transcription machinery, the components of the core transcriptional apparatus and the chromatin DNA template are subject to regulation. For instance, a variety of covalent histone and DNA modifications can influence whether a chromatin template is programmed to be transcriptionally active or silent (Strahl and Allis 2000; Berger 2002; Geiman and Robertson 2002). Also, numerous chromatin remodeling/nucleosome mobilizing activities catalyze the ATP-dependent deposition, removal, or sliding of nucleosomes to generate DNA templates that are accessible to the transcription machinery (Becker and Horz 2002). An enormous family of DNA sequence-specific transcriptional activators working in concert with various coregulatory factors drive the formation of active transcription initiation complexes

${ }^{1}$ Corresponding author.

EMAIL jmlim@uclink4.berkeley.edu; FAX (510) 643-9547.

Article and publication are at http://www.genesdev.org/cgi/doi/10.1101/ gad.1099903.
(Lemon and Tjian 2000; Malik and Roeder 2000; Naar et al. 2001). It now seems clear that this highly regulated and coordinated assembly of active transcription complexes requires the interplay of a large number of transcription factors, multiple core promoter elements, and chromatin remodeling and modifying factors to properly position the preinitiation complex (PIC) at the start site of RNA synthesis (Burley and Roeder 1996; Roeder 1996; Lemon and Tjian 2000; Fig. 1).

Until recently, the general transcription machinery that makes up the PIC was thought to be largely invariant when compared among different cell types within an organism. Although a large number of tissue-specific transcription factors have been described that control expression of cell type-specific genes, most of these have been enhancer-binding transcription factors. However, contrary to expectations, several studies have identified cell type-specific components of the core transcription machinery. Subsequently, an increasing number of cell type-specific and gene-selective homologs of basal transcription factors have been identified in metazoan organisms, including additional members of the TATA boxbinding protein (TBP) family such as TBP-related factors (TRFs) as well as numerous tissue-specific homologs of TBP-associated factors (TAFs). It now seems apparent that multicellular organisms have evolved cell type-specific components of the general transcriptional apparatus as a mechanism to accommodate more complex programs of tissue-specific and gene-selective transcription. Intriguingly, many of these functionally specialized core promoter factors are subunits of TFIID. This central component of the PIC harbors several transcription factors directly involved in recognizing and binding core promoter elements such as the TATA box, Initiator (Inr), and downstream promoter element (DPE). These findings suggest that metazoans have evolved functionally specialized transcription initiation complexes and promoter-selective modules, which might serve as eukaryotic $\sigma$ factors (Gross et al. 1998; Losick 1998).

In this review, we will summarize some recent advances in our understanding of how these specialized transcription initiation complexes might mediate pro- 
Figure 1. The transcription apparatus is a multilayered ensemble of multisubunit complexes. The transcription machinery is responsible for the decoding and expression of genes in a regulatory network composed of various coregulatory complexes that are interconnected to control RNA synthesis from a given promoter. This includes covalent modifications of histone tails and DNA that can influence whether a chromatin template is programmed to be transcriptionally active or silent. Chromatin remodeling activities catalyze the ATP-dependent deposition, removal, or sliding of nucleosomes to create a DNA template that is accessible to transcription factors. Activated transcription is controlled by coregulatory coactivator complexes that mediate activation of transcription by activators located in a promoter-proximal position (PA) or many kilobases away in a promoter-distal (DA) position. Also, the composition and sequence of the core promoter directs the formation of the preinitiation complex and defines the start site of transcription. This involves the interplay of basal transcription factors (TFIIA, TFIIB, TFIID, TFIIE, TFIIF, and TFIH) and RNA polymerase with distinct core promoter elements like TATA box (TATA), Initiator (Inr), and downstream promoter element (DPE) to position the preinitiation complex (PIC).

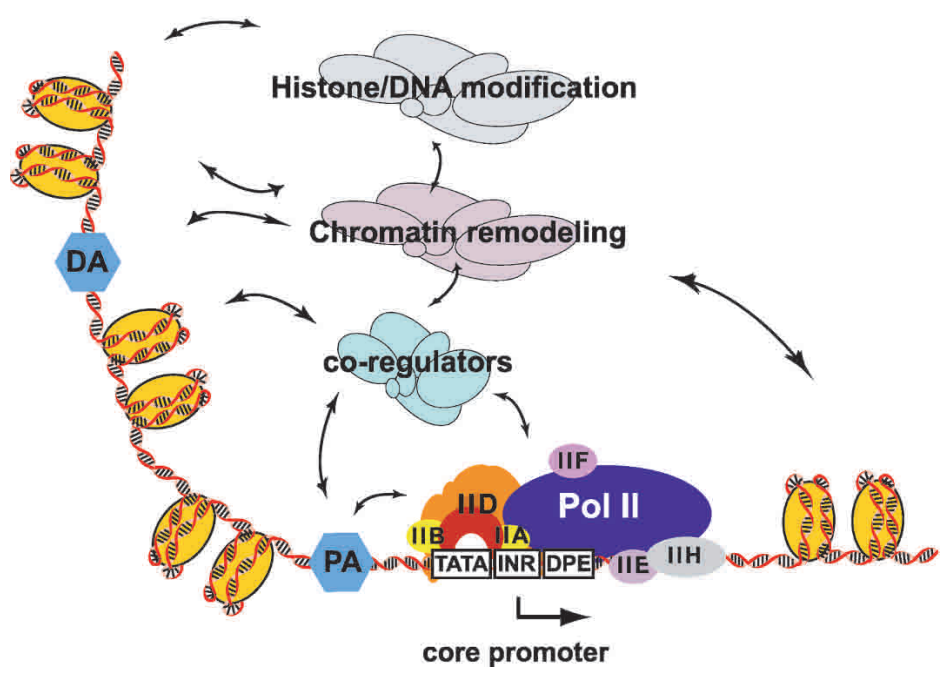

moter selectivity. We will also discuss how tissue-specific and gene-selective core promoter complexes might regulate coordinatedly expressed genes responsible for executing essential biological functions such as DNA replication, organ development, and germ cell maturation.

\section{The family of TRFs}

The TBP has been established as a universal and essential transcription factor required by all three RNA polymerases to recognize promoters and initiate transcription in eukaryotic organisms (Hernandez 1993; Burley and Roeder 1996). This includes the transcription of protein-encoding genes by RNA polymerase II (Pol II) as well as ribosomal RNAs and small, stable RNAs transcribed by RNA polymerases I (Pol I) and RNA polymerase III (Pol III), respectively. Indeed, from human to yeast, TBP was shown to be a component of three different multisubunit transcription initiation complexes including SL1, TFIID, and TFIIIB. These initiation complexes are involved in directing transcription by RNA polymerases I, II, and III, respectively (Dynlacht et al. 1991; Comai et al. 1992; Sharp 1992; Hernandez 1993; Burley and Roeder 1996; Fig. 2A). In each class of transcription initiation complexes, TBP makes specific contacts with associated proteins and/or promoter DNA to help nucleate assembly of the appropriate RNA polymerase initiation complex. In the case of RNA pol II, TFIID consists of TBP and several TBP-associated factors $\left(\mathrm{TAF}_{\mathrm{II}} \mathrm{s}\right)$, which can directly bind to specific core promoter elements such as the TATA box, Inr, and DPE (Butler and Kadonaga 2002) to form an active PIC containing RNA pol II and the general transcription factors TFIIA, TFIIB, TFIIE, TFIIF, and TFIIH (Figs. 1, 2A). It is now well established that the sum of these interactions between initiation factors and different core promoter elements defines the start site of transcription and may also modulate the strength of a particular promoter (Smale 2001). When this rather elaborate PIC was first identified, it did not seem necessary to invoke the existence of additional gene-selective or cell type-specific subunits of an already complex multisubunit basal transcription apparatus. However, a novel gene encoding a protein closely related to but distinct from TBP was identified in Drosophila and subsequently named TBP-related factor 1, TRF1 (Crowley et al. 1993).

\section{TRF1 has promoter-selective properties}

Drosophila embryo in situ staining experiments revealed that TRF1 is most highly expressed in the nervous system and gonads (Crowley et al. 1993; Hansen et al. 1997). Biochemical studies confirmed that TRF1, like TBP, can interact with TFIIA and TFIIB, bind to TATA box DNA, and direct RNA pol II-specific transcription in vitro (Hansen et al. 1997). Like TBP, TRF1 was found by biochemical fractionation to be part of large multisubunit complexes (Fig. 2B). Importantly, Drosophila polytene chromosome staining revealed that, unlike TBP, TRF1 is only associated with a rather limited set of loci, which are distinct from those associated with TBP (Hansen et al. 1997). These findings, taken together with the somewhat tissue-restricted expression pattern of TRF1, suggested that TRF1 may be a functional homolog of the prototypic core transcription factor TBP that may have diversified to direct tissue-specific or gene-selective transcription in Drosophila. Chromatin immunoprecipitation (ChIP) experiments subsequently identified several Drosophila genes containing a TRF1-responsive promoter (Holmes and Tjian 2000). Biochemical characterization revealed that TRF1 can preferentially bind and 


\section{Human and mouse}
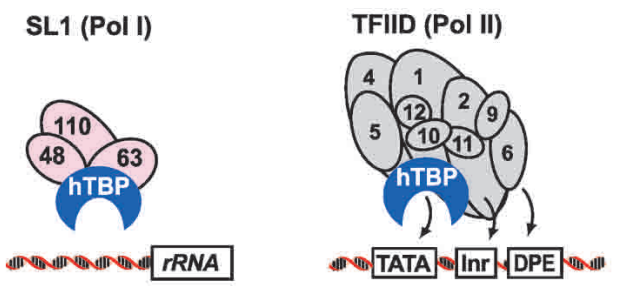

TFIIIB (Pol III)

hTRF2:TFIIA (Pol II)

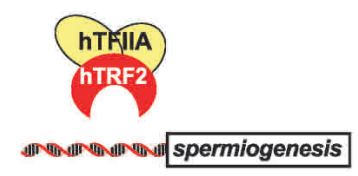

B

Drosophila

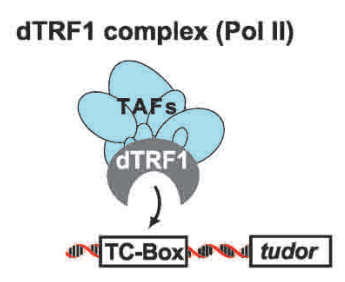

dTRF1:BRF (Pol III)

dTRF2 complex (Pol II)

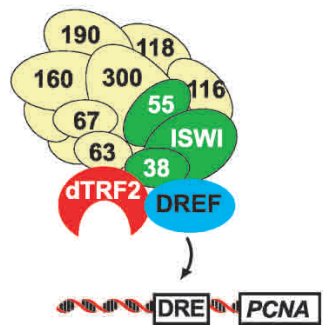

Figure 2. TBP and TBP-related factors (TRFs) are components of distinct transcription initiation complexes. (A) TBP is a component of all three transcription initiation complexes SL1, TFIID, and TFIIIB, representing RNA polymerase I (Pol I), RNA polymerase II (Pol II), and RNA polymerase III (Pol III). TBP is associated with three TBP-associated factors $\left(\mathrm{TAF}_{\mathrm{I}} \mathrm{S}\right.$; molecular weight in kilodaltons is given) in SL1 to initiate transcription of rRNA genes. In TFIID, TBP is associated with 12-15 $\mathrm{TAF}_{\mathrm{II}} \mathrm{s}$ to initiate transcription of protein-encoding genes. TBP binds the TATA box and several $\mathrm{TAF}_{\mathrm{II}} \mathrm{S}$ can specifically recognize additional core promoter elements such as the Initiator (Inr, $\mathrm{TAF}_{\mathrm{II}} 2$ ) and the downstream promoter element (DPE, $\mathrm{TAF}_{\mathrm{II}} 6$ ). TBP associates with BRF to form TFIIIB to initiate transcription of small RNAs. Human TBPrelated factor 2 (hTRF2) was purified in a stable complex with the general transcription factor TFIIA. Mouse TRF2 is involved in the transcription of spermiogenesis genes. (B) Drosophila TRF1 was isolated in a complex with TRF1-associated factors (TAFs). TRF1 recognizes a TC-rich region (TC box) upstream of the TRF1-dependent transcription start site of the tudor gene. Drosophila TRF1 is also associated with BRF and is required for Pol III transcription of small RNAs. Drosophila TBP-related factor 2 (dTRF2) is part of a multisubunit complex composed of the DNA replication-related element (DRE)-binding factor (DREF), subunits of the chromatin-remodeling complex NURF, and additional associated factors. The DREFcontaining TRF2 complex functions as a promoterselectivity factor to control transcription of cell proliferation genes such as PCNA. direct transcription from one of two tandem promoters of the tudor gene, which is involved in Drosophila male fertility. The TRF1-binding region was determined by deoxyribonuclease footprinting to be a TC-rich region (TC box) rather than a TATA box located at position -25 relative to the TRF1-dependent transcription start site (Holmes and Tjian 2000; Fig. 2B). This study also revealed that the tudor gene has a second TBP/TFIID-dependent promoter that is not responsive to TRF1. Such an arrangement of tandem promoters provided an interesting mechanism by which an alternative core promoter factor like TRF1 could expand the programs of gene expression for a subset of genes.

Endogenous TRF1 had been isolated in a complex with other associated proteins (putative TRF1-associated factors; Fig. 2B). This finding indicated that the ability of TRF1 to recognize specific core promoter sequences might not be solely due to the intrinsic DNA-recognition properties of TRF1. Instead, the promoter specificity of TRF1 could be significantly influenced by its associated subunits much the same way that TBP collaborates with TAFs in TFIID or SL1 to direct either RNA pol II or I transcription, respectively (Fig. 2A). It therefore seemed reasonable to postulate that the promoter selectivity properties of TRF1 might depend in part on its associated factors and that a more exhaustive analysis of TRF1 might unmask additional target genes.

\section{A TRF1:BRF complex directs RNA pol III transcription}

Given this new context, it was interesting to find that TRF1 localized not only to various genes linked to male sterility or neuronal functions, but polytene chromosome staining studies also mapped TRF1 to many tRNA 
and 5S RNA gene loci (Hansen et al. 1997). This intriguing finding suggested that TRF1, like TBP, could be involved in mediating both RNA pol II and III transcription in Drosophila.

To test this hypothesis Takada et al. (2000) developed a Drosophila RNA pol III in vitro transcription system and asked the simple question: Is TRF1, TBP, or both required for RNA pol III transcription? Surprisingly, biochemical complementation studies revealed that TRF1 rather than TBP plays a major role in RNA pol III transcription (Takada et al. 2000). This was an unexpected finding because in human, yeast, and most other systems that had been studied, TBP together with BRF are essential components of the RNA pol III transcription initiation factor TFIIIB (Geiduschek and Kassavetis 1995; Fig. 2A). Subsequent coimmunoprecipitation experiments led to the identification of Drosophila BRF as a TRF1associated factor that directs RNA pol III transcription in Drosophila cells (Fig. 2B). BRF forms a stable complex with TRF1 rather than TBP and this TRF1:BRF complex is an essential component of the RNA pol III transcription apparatus (Takada et al. 2000). Indeed, a major fraction of TRF1 in Drosophila SL2 cells is complexed with BRF, whereas no significant amounts of a TBP:BRF complex could be detected. These findings provided further evidence that the Drosophila transcription machinery had diversified and evolved a unique Pol III transcription system involving TRF1. However, because TRF1 is differentially expressed in Drosophila cell types, it was not surprising to find that the proportion of TRF1 associated with BRF differs somewhat when comparing SL2 cells and developing Drosophila embryos (Takada et al. 2000). This is consistent with the observation that TRF1 can also operate as a promoter-selective regulator of RNA pol II transcription (Holmes and Tjian 2000). These dual functions of TRF1 are likely to depend on its association with different subunits, adding further diversity to the function of this core transcription factor (Fig. 2B). An advantage of this strategy is that metazoan organisms like Drosophila can exploit the specialized properties of basal transcription factor homologs such as TRF1 to mix and match with additional factors to expand their functional specificity, perhaps modulating potential tissuespecific and/or gene-selective activities.

\section{TBP-related factor 2 (TRF2): another member of the TBP family}

The discovery of TRF1 led to a search for additional alternative core promoter complexes. These studies were largely carried out in flies, worms, frogs, and mammals because TRFs were not found to be present in yeast or other unicellular eukaryotes.

Database homology searches quickly led to the identification of a third member of the TBP family in Drosophila called TBP-related factor 2 (TRF2; Rabenstein et al. 1999|, which was present in a large variety of other metazoan organisms from Caenorhabditis elegans and Xenopus to mouse and man (Dantonel et al. 1999; Rabenstein et al. 1999; Fig. 3). Because TRF2 has been in-
A
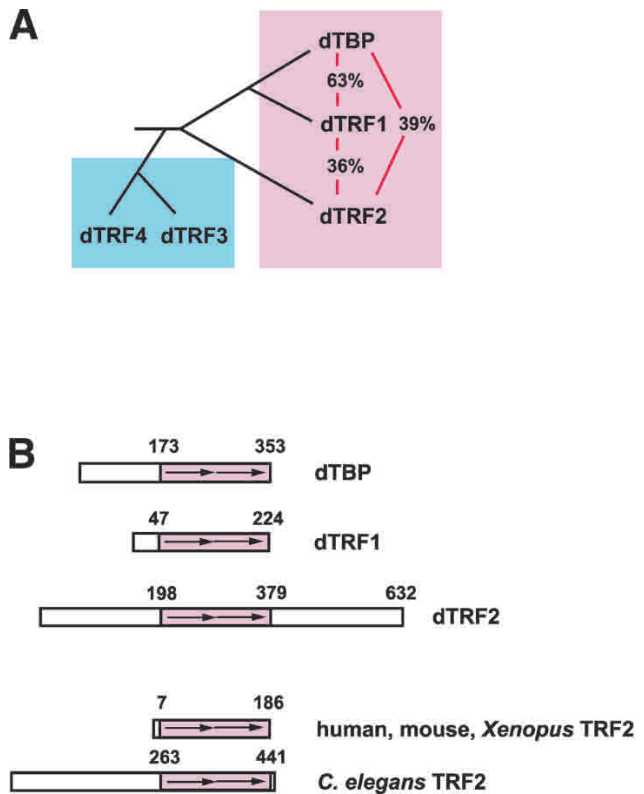

Figure 3. The TBP family: TATA box-binding protein (TBP) and TBP-related factors (TRFs). Sequence comparison and phylogenetic analysis revealed that TRF2s constitute a distinct family of TBP-related factors in metazoan organisms. (A) Notably, the Drosophila TBP family is very diverse. TRF1 is a unique member of the TBP family discovered only in Drosophila and is the closest relative of TBP discovered so far. TRF2 is the third member of the TBP family and is more closely related to TBP than to TRF1. This suggested that TRF2 diverged from TBP and that TRF1 evolved later from TBP. Recently, two more distantly related TBP-related factors, TRF3 and TRF4, have been discovered forming a distinct fourth family of TBP-related factors in Drosophila. (B) Drosophila TRF1 and all TRF2s share a conserved bipartite core repeat with TBP that represents the DNAbinding domain in TBP (repeat arrow). Although this core domain is highly conserved throughout the TBP family, TRF2s differ significantly in size and sequence, particularly within their $\mathrm{N}$-terminal and $\mathrm{C}$-terminal domains, suggesting that these unique domains contribute to the functional diversity among TBP-related factors.

dependently isolated from several different organisms it has variously been called TRF (Maldonado 1999), TBPlike factor (TLF; Dantonel et al. 1999; Kaltenbach et al. 2000; Veenstra et al. 2000), or TBP-like protein (TLP; Ohbayashi et al. 1999a,b). To avoid confusion, we will use the TRF2 terminology for this protein family of factors based on the nomenclature of the founding member TRF1 (Crowley et al. 1993).

A sequence comparison and phylogenetic analysis of the various TRF2s in metazoan organisms established that TRF2s constitute a family of TBP-related factors more closely related to TBP than to TRF1, the closest relative of TBP isolated thus far (Rabenstein et al. 1999; Dantonel et al. 2000; Fig. 3A). Both TRF1 and the TRF2s share a conserved bipartite repeat domain common to all TBPs that functions as the DNA-binding domain in TBP (Fig. 3B). More than $80 \%$ of the amino acid residues in TBP involved in DNA binding (Kim et al. 1993) are conserved in TRF1 (Rabenstein et al. 1999; Dantonel et al. 
2000). As expected, TRF1 can bind to canonical TATA boxes in addition to TC boxes (Crowley et al. 1993; Hansen et al. 1997; Holmes and Tjian 2000). Although this DNA-binding domain is highly conserved in TRF2s (50\% identity) a detailed sequence comparison revealed that key amino acids crucial for the interaction of TBP with the minor groove of the TATA DNA template have diverged in TRF2s (Dantonel et al. 2000). This suggested that TRF2s might not recognize TATA boxes and may bind DNA with a completely different specificity. This is consistent with a majority of reports indicating that recombinant TRF2s failed to bind canonical TATA boxes (Moore et al. 1999; Rabenstein et al. 1999; Teichmann et al. 1999). Although the TRF2s may have a diverged DNA recognition domain, they have conserved binding motifs for TFIIA and TFIIB and can interact with these two essential components of the PIC (Rabenstein et al. 1999; Teichmann et al. 1999) suggesting a possible role for TRF2s in RNA pol II transcription.

\section{TRF2s are required for embryonic development and differentiation}

The first clues concerning the biological function of TRF2s came from depletion studies in C. elegans and Xenopus using RNA interference (RNAi) or antisense oligonucleotide mediated turnover of TRF2 (Dantonel et al. 2000; Kaltenbach et al. 2000; Veenstra et al. 2000). The depletion of TRF2 in worms and frogs resulted in early embryonic developmental arrests. An analysis of gene expression patterns revealed that these arrested embryos failed to express certain differentiation markers and displayed a generalized decrease in Pol II transcription. Interestingly, depletion of TRF2 also caused the disregulation of developmental genes at very early stages of embryogenesis (Dantonel et al. 2000). These studies suggested that TRF2 may be required for transcription of some key developmental genes, whereas other genes are unaffected in TRF2-RNAi-treated embryos. Thus, TRF2, like TRF1, may be required to mediate transcription of only a limited subset of genes (Kaltenbach et al. 2000). Additional antisense studies in Xenopus and zebrafish revealed that there is a complementary requirement for TBP and TRF2 in directing expression of some genes. This finding strengthens the notion that TBP and TRF2 can function differentially to control transcription of specific gene sets in animal cells (Veenstra et al. 2000; Muller et al. 2001).

\section{Murine TRF2 is required for spermiogenesis}

Northern blot analysis had indicated that human TRF2 is differentially expressed in a tissue-specific fashion with the highest expression levels observed in testis (Rabenstein et al. 1999). To elucidate its potential biological function, $\mathrm{TRF}^{-/-}$knockout $(\mathrm{KO})$ mice were generated by homologous recombination (Martianov et al. 2001; Zhang et al. 2001). TRF2-deficient mice are viable; however, mutant male mice are sterile because of a late ar- rest in spermiogenesis. This suggested that murine TRF2, unlike frog or fish TRF2, may not be required for embryonic development but is essential for spermiogenesis. Northern blot and RT-PCR analysis further confirmed that TRF2-deficient mice display an altered expression pattern of testis-specific genes involved in spermiogenesis consistent with the notion that TRF2 might be specifically involved in the transcriptional regulation of sperm formation (Martianov et al. 2001; Zhang et al. 2001).

The apparent testis-specific function of murine TRF2 is in stark contrast to the strong early embryonic phenotypes seen in C. elegans, Xenopus, and zebrafish. Although the members of the TRF2 family are closely related and share a highly conserved putative DNA-binding motif, they vary considerably in size and domain architecture (Dantonel et al. 1999; Rabenstein et al. 1999; Fig. 3B). Notably, the mammalian TRF2 is relatively small and consists largely of the bipartite core repeat, whereas TRF2 in C. elegans and Drosophila has extended and unique $\mathrm{N}$-terminal and C-terminal domains (Dantonel et al. 1999; Rabenstein et al. 1999). This suggested that different species may have evolved specialized TRF2s to execute specific biological functions. In this context it is interesting to note that the vertebrate-specific N-terminal domain of TBP is required for placental development in mice (Hobbs et al. 2002). Despite these in vivo studies, the mechanisms by which TRF2s control transcription remained elusive, and specific TRF2 target genes were yet to be conclusively identified.

\section{TRF2 directs promoter-selective gene expression in Drosophila}

Drosophila TRF2, like TBP and TRF1, was shown to interact with the basal transcription factors TFIIA and TFIIB. However, dTRF2 failed to bind to DNA containing canonical TATA boxes (Rabenstein et al. 1999). Interestingly, TRF2 is associated with loci on Drosophila chromosomes that are distinct from both TBP and TRF1 (Rabenstein et al. 1999). This suggested that TRF2 may direct promoter specificity and perhaps coordinately regulate a select subset of target genes. Size exclusion chromatography indicated that Drosophila TRF2 is likely part of a macromolecular complex. The diverged $\mathrm{N}$-terminal and C-terminal domains of TRF2 also suggested that TRF2 may be associated with a novel set of proteins that are distinct from TBP- and TRF1-associated factors (Goodrich and Tjian 1994; Hansen et al. 1997. Takada et al. 2000; Fig. 3B). Antibody affinity purification ultimately led to the identification of Drosophila TRF2-containing complexes that possess components of the NURF chromatin remodeling complex, the DNA replication-related element (DRE)-binding factor DREF, and other proteins that might be involved in chromatin transactions (Hochheimer et al. 2002; Fig. 2B). The biochemical characterization of a TRF2-containing complex subsequently led to the identification of TRF2-specific promoters and revealed how TRF2 might operate to ex- 
ecute transcriptional specificity. An important clue was the identification of DREF as a TRF2-associated factor. DREF had been previously reported to be the cognate binding factor of the promoter proximal DRE, a DNA element that is involved in the regulation of cell cycle and cell proliferation genes (e.g., PCNA and DNApol 180; Hirose et al. 1993). Thus, TRF2 might cooperate with DREF to function as a metazoan promoter-selectivity factor.

A combination of in vitro and cell-based assays established that the DREF-containing TRF2 complex directs core promoter recognition of the proliferating cell nuclear antigen (PCNA) gene (Hochheimer et al. 2002). In a manner reminiscent of the TRF1-responsive tandem promoters, the TRF2-responsive PCNA gene also uses two tandem core promoters, which are separated by 63 bp (Fig. 4). The DREF/TRF2 complex selectively initiates transcription from Promoter 2, which harbors the DREFbinding site (DRE), whereas Promoter 1 is stimulated by TBP/TFIID (Hochheimer et al. 2002; Fig. 4). Gene expression analysis identified several additional TRF2-responsive target genes involved in DNA replication and cell proliferation that contain promoter-proximal DRE elements (Hochheimer et al. 2002). Thus, Promoter 2 of the PCNA gene may represent a class of promoters that harbor the DRE element. Notably, a computational analysis of core promoter elements in the Drosophila genome independently identified the DRE as one of the most conserved potential core promoter elements, together with well-established cis-controlling sequences such as the TATA box, Inr, and DPE (Ohler et al. 2002). Furthermore, transcription profiling experiments combining fluorescence-activated cell-sorting (FACS) and serial analysis of gene expression (SAGE) suggested that the DRE is involved in the control of eye development, in particular at the transition from cell proliferation to terminal differentiation (Jasper et al. 2002). Taken together, these data established that TRF2 can function as a core promoter-selectivity factor responsible for coordinately regulating transcription of a subset of genes in Drosophila.

\section{Tandem promoters expand metazoan gene networks}

Interestingly, the promoter-selective properties of TRF1 and TRF2 revealed that both of these TBP-related factors direct transcription at genes with tandem core promoters that are selectively used by TBP/TFIID or by TRF1/ TRF2. Such an arrangement of multiple distinct core promoters could represent one mechanism evolved by metazoan organisms to expand their repertoire of gene expression networks. Instead of duplicating genes, tandem core promoters would allow a given cell type to regulate a single gene that may be expressed at different times during development or in different cell types by using functionally specialized transcription initiation complexes. At present, the functional specificities of the tandem promoters driving the tudor (TRF1) and PCNA (TRF2) genes remain unclear. We speculate that transcription initiation at these alternate promoters could trigger previously uncharacterized regulatory events required to establish transcriptionally competent gene loci that are differentially regulated possibly in a cell typespecific or temporally controlled manner.

\section{Potential multiple functions of the TRF2-containing complexes}

Drosophila TRF1 and TRF2 have been isolated as part of multisubunit complexes composed of various proteins that can modulate the functional specificity of these transcription initiation complexes (Hansen et al. 1997; Holmes and Tjian 2000; Takada et al. 2000; Hochheimer et al. 2002). By contrast, mammalian TRF2 has thus far only been purified in a stable complex with TFIIA (Teichmann et al. 1999; Fig. 2A) and the mechanism by which mammalian TRF2 directs RNA pol II transcription is still unclear. In vitro transcription experiments using recombinant human TRF2 or an endogenous human TRF2-TFIIA complex revealed that hTRF2 cannot replace TBP or TFIID in basal or activated transcription at various Pol II promoters (Teichmann et al. 1999). However, addition of recombinant hTRF2 to in vitro transcription reactions inhibits TBP-dependent transcription and this repression can be alleviated by the addition of TFIIA. This suggested that the observed repression by hTRF2 may involve sequestering of TFIIA and/or other components of the basal transcription machinery (Moore et al. 1999; Teichmann et al. 1999). It is thus likely that mammalian TRF2 requires additional uncharacterized factors to execute its transcriptional specificity. It is noteworthy in this context that the hu-

Figure 4. Promoter-selective transcription at the PCNA tandem core promoters. The DREF-containing TRF2 complex contains components of the NURF chromatin remodeling complex (ISWI, NURF-55/p55, NURF-38/p38) as well as the DNA replication-related element (DRE)-binding factor DREF, and additional proteins that might be involved in mediating additional chromatin transactions (molecular weight in kilodaltons is given). The DREF/TRF2 complex initiates transcription at the DRE-containing Promoter 2, whereas Promoter 1, which is located $63 \mathrm{bp}$ downstream of Promoter 2, is initiated by TBP/TFIID.

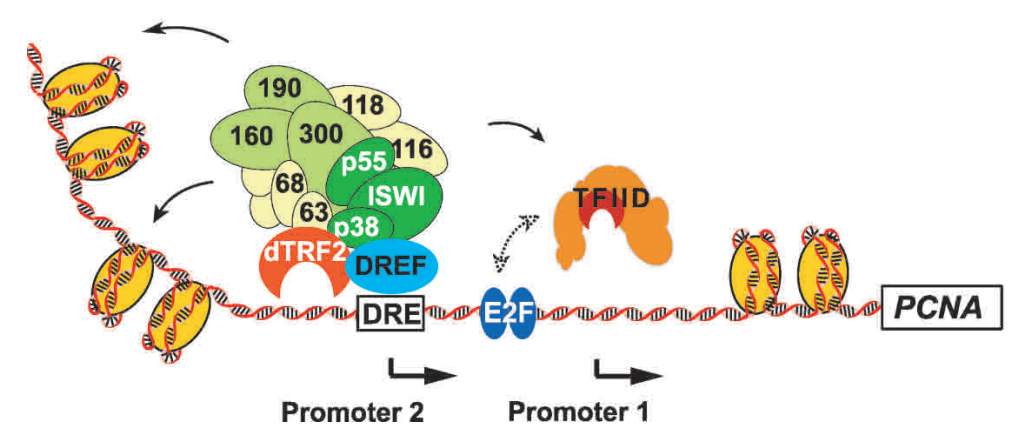


man genome encodes a TFIIA-like factor ALF (or TFIIA $\tau$ ) that is highly expressed in testis. Like TFIIA, ALF promotes TBP binding to DNA and can replace TFIIA in an in vitro transcription system (Upadhyaya et al. 1999, 2002; Ozer et al. 2000). However, a role for ALF in spermatogenesis has not been observed to date and there is little evidence that ALF can replace TFIIA to direct tissue-specific transcription.

\section{Tissue-specific TAFs and functionally specialized TFIID complexes}

The eukaryotic TFIID complex composed of the TBP and 12-15 $\mathrm{TAF}_{\mathrm{II}} \mathrm{s}$ has emerged as one of the central components of a highly versatile transcription apparatus (Fig. 5, center). This multifunctional transcription complex has thus far been shown to be responsible for core promoter recognition, coactivator function, catalysis of protein modification, and targeting to specifically acetylated nucleosomes. For example, TFIID functions to recognize the core promoter and to provide a "landing pad" upon which the other transcription factors and RNA pol II can assemble to form the PIC (Burley and Roeder 1996; Albright and Tjian 2000). Individual $\mathrm{TAF}_{\mathrm{II}} \mathrm{S}$ can recognize specific core promoter elements such as the Inr and the DPE in conjunction with TBP binding to the TATA element to position the PIC and define the start site of transcription (Albright and Tjian 2000; Butler and Kadonaga 2002; Fig. 5, center). $\mathrm{TAF}_{\mathrm{II}} \mathrm{S}$ also play an important role in transcriptional activation by providing various coactivator interfaces targeted by different activation domains of enhancer-binding transcription factors (Verrijzer and Tjian 1996). Remarkably, recent studies have documented the ability of TAF1 (formerly $\mathrm{TAF}_{\text {II }} 250$ ) bromodomains to specifically recognize and bind to diacetylated histone $\mathrm{H} 3$ and $\mathrm{H} 4 \mathrm{~N}$-terminal tails.

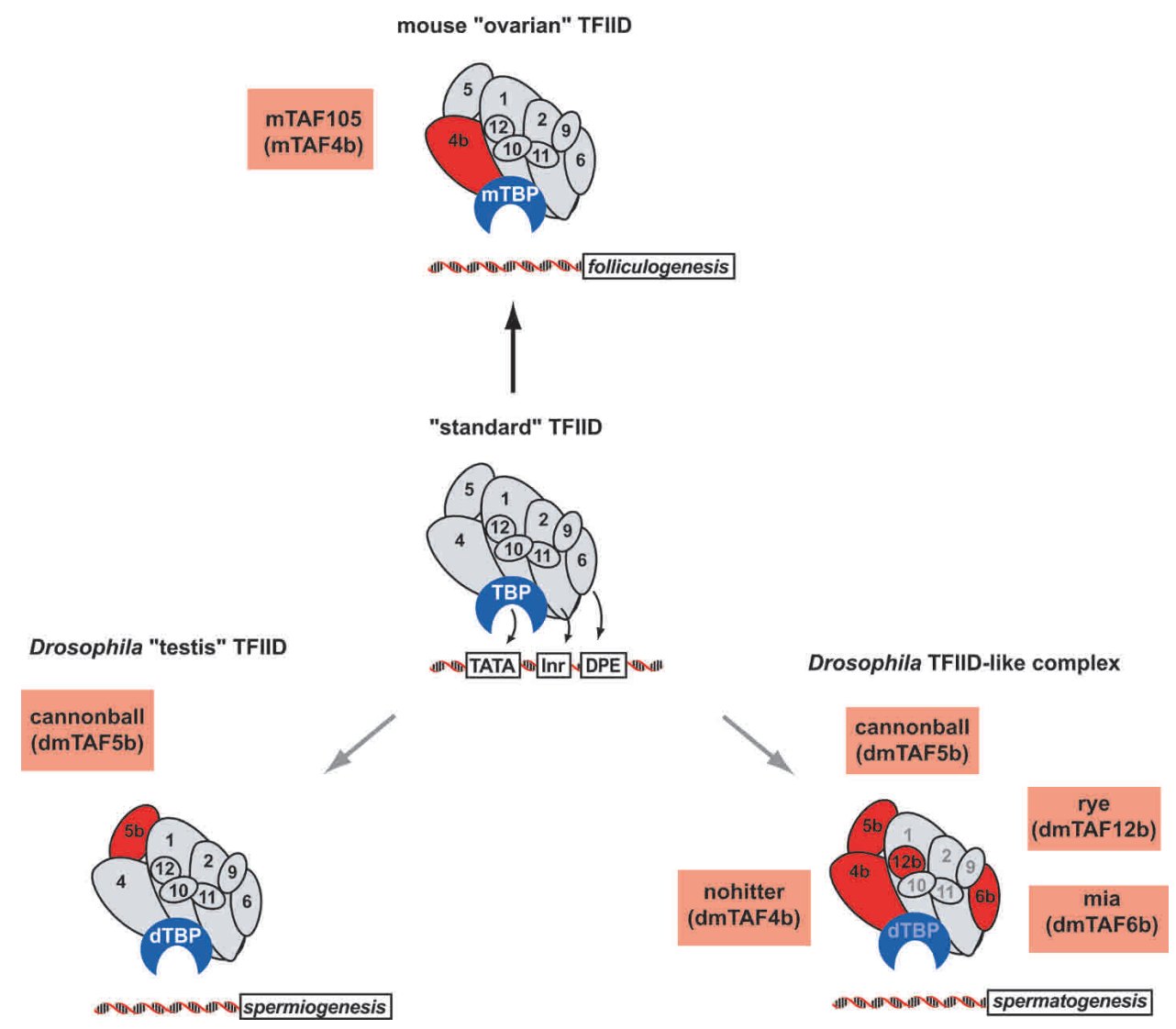

Figure 5. Functionally specialized TFIID complexes govern tissue-specific transcription. The "standard" TFIID is composed of the TATA box-binding protein $(\mathrm{TBP})$ and $\mathrm{TBP}$-associated factors $\left(\mathrm{TAF}_{\mathrm{II}} \mathrm{S}\right)$ and initiates transcription of many protein-encoding genes (center). TBP binds the TATA box and some $\mathrm{TAF}_{\mathrm{II}} \mathrm{S}$ selectively recognize core promoter elements such as the Initiator (Inr) and the downstream promoter element (DPE; center). Mouse TAF4b $\left(\mathrm{mTAF}_{\mathrm{II}} 105\right)$ is specifically expressed in ovarian granulosa cells and is part of a functionally specialized "ovarian" TFIID complex regulating ovary-specific folliculogenesis genes (top). Drosophila cannonball (can) is a homolog of dmTAF5 ( $\left.\mathrm{dTAF}_{\mathrm{II}} 80\right)$. Expression of cannonball is testis-specific in primary spermatocytes, whereas dmTAF5 is ubiquitously expressed. Cannonball is required for transcription of spermatid differentiation genes and might replace dmTAF5 in a testis-specific TFIID complex (bottom left). Drosophila encodes three additional testis-specific TAF II $_{\text {iI }}$ isoforms. Nohitter (nht; related to dmTAF4/dTAF 1110 ), ryan express (rye; related to dmTAF12/dTAF $30 \mathrm{a})$, and meiosis I arrest (mia; related to $\mathrm{dmTAF}_{\mathrm{II}} \mathrm{b} / \mathrm{dTAF}_{\mathrm{II}} 60$ ) could be part of a testis-specific TFIID-like transcription initiation complex composed of several testis-specific TAF isoforms (bottom right). 
This unexpected finding opens the possibility that TFIID can mediate formation of a PIC even at nucleosome-occupied promoters (Jacobson et al. 2000). Several studies have reported that TAF1 can catalyze various enzymatic activities including kinase, acetyl transferase, and ubiquitin ligase (Dikstein et al. 1996a; Mizzen et al. 1996; O'Brien and Tjian 1998; Pham and Sauer 2000). Thus, the TBP/TAF complex appears to be a multifunctional complex that may serve as a key integrator of molecular signals to and from the central transcriptional machinery.

Whole genome analysis of Drosophila, human, C. elegans, and yeast have led to the identification of many TAF orthologs and paralogs including some that display cell type-specific expression patterns in a variety of tissues (Aoyagi and Wassarman 2000; Veenstra and Wolffe 2001). This suggested that $\operatorname{TAF}_{I I} \mathrm{~S}$ may contribute to tissue-specific gene regulation in metazoan organisms. Despite the large and still growing number of reports of $\mathrm{TAF}_{\mathrm{II}} \mathrm{S}$ with a differential pattern of expression only a few variant $\mathrm{TAF}_{\mathrm{II}} \mathrm{s}$ have been studied in detail to determine whether they are indeed involved in tissue-specific gene expression.

\section{Tissue-selective properties of mammalian TAF4b}

The first cell type-specific subunit of TFIID, hsTAF4b (formerly called $\mathrm{TAF}_{\text {II }} 105$ ), was discovered in a highly differentiated human B cell line (Dikstein et al. 1996b). Analysis of the primary amino acid sequence revealed that hsTAF4b is closely related to the more broadly expressed human hsTAF4 (formerly TAF II $_{130}$; Tanese et al. 1996) and its Drosophila homolog dmTAF4 (TAF 110 ; Hoey et al. 1993). Expression analysis revealed that a mouse homolog of TAF4b is also expressed in a tissuespecific fashion with the highest expression detected in testis and ovaries (Freiman et al. 2001). Importantly, mouse TAF4b was shown to be an intrinsic subunit of a large complex containing both mTAF1 $\left(\mathrm{mTAF}_{\mathrm{II}} 250\right)$ and mTBP in ovarian extracts, thus confirming that TAF4b is indeed a subunit of an ovary-specific TFIID (Freiman et al. 2001; Fig. 5, top). The analysis of TAF4b-deficient $\left(\mathrm{TAF}_{\mathrm{II}} 105^{-1-} \mathrm{KO}\right)$ mice revealed that females lacking TAF4b are viable but infertile because of a defect in ovarian folliculogenesis. This correlated well with the restricted expression pattern of TAF4b in the granulosa cells of the ovarian follicle (Freiman et al. 2001). In order to probe potential molecular mechanisms underlying the folliculogenesis defect and to identify possible TAF4b target genes, a microarray-based gene expression profiling experiment was performed. This study revealed that a subset of genes involved in female reproduction are specifically down-regulated in TAF4b-deficient ovaries (Freiman et al. 2001). This strongly suggested that TAF4b is a cell type-specific component of the mammalian transcription machinery that mediates expression of a subset of genes required for proper folliculogenesis.

Although the TAF4b KO mice did not display any obvious defect in B-cell development or function (Freiman et al. 2002), spleen B cells do express elevated levels of TAF4b. This is consistent with the original isolation of
TAF4b from an established human B-cell line (Dikstein et al. 1996b). Thus, it seems likely that TAF4b also functions in B cells as well as in gonads. Indeed, several studies have demonstrated that TAF4b interacts with regulators of B-cell transcription, including members of the NF- $\kappa \mathrm{B} /$ Rel family of transcription factors and OCA-B, a B-cell-specific coactivator (Yamit-Hezi and Dikstein 1998; Wolstein et al. 2000; Yamit-Hezi et al. 2000). Interestingly, chromatin immunoprecipitation analysis revealed that both hTAF4b and hTBP colocalize to promoters of NF-кB-hTAF4b-regulated genes (Ainbinder et al. 2002), indicating that the functionally specialized TAF4b-containing TFIID complex may interact with specific core promoter elements.

\section{Cannonball is required for spermatogenesis in Drosophila}

Although TAF4b was the first tissue-specific TAF to be identified, it now seems apparent that the diversification of specialized $\mathrm{TAF}_{\mathrm{II}} \mathrm{s}$ may be more prevalent than originally anticipated. A systematic and elegant genetic study in Drosophila identified at least four testis-specific TAF isoforms. No hitter (nht) is related to dmTAF4 (formerly dTAF $_{\text {II }} 110$ ), cannonball (can) to dmTAF5 (formerly dTAF $_{\text {II }} 80$ ), ryan express (rye) to dmTAF12 (formerly $\operatorname{dTAF}_{I I} 30 a$ ), and meiosis I arrest (mia) to dmTAF6 (formerly dTAF ${ }_{\text {II }} 60$; Aoyagi and Wassarman 2000; Hiller et al. 2001; Veenstra and Wolffe 2001). Expression of cannonball is stage- and tissue-specific and restricted to primary spermatocytes, whereas its prototypic counterpart dmTAF5 is ubiquitously expressed (Hiller et al. 2001). Cannonball is required for expression of several spermatid differentiation genes (White-Cooper et al. 1998) and for germ cell development in male flies (Hiller et al. 2001). In cannonball null mutants, the transcription of specific target genes is significantly reduced and, consequently, meiotic cell cycle progression is blocked, resulting in a failure of spermatid differentiation (Hiller et al. 2001). These studies strongly suggest that cannonball is required in vivo for the transcription of stage- and tissuespecific target genes required for proper male fly gametogenesis.

It is currently unclear whether cannonball or any other of the testis-specific TAF isoforms interact with the prototypic $\mathrm{TAF}_{\mathrm{II}} \mathrm{s}$ to form a functionally specialized TFIID complex that initiates transcription at a select subset of gametogenesis promoters (Fig. 5, bottom left). It is striking, however, that Drosophila has at least four testisspecific isoforms of $\mathrm{dTAF}_{\mathrm{II}} \mathrm{s}$. It is thus tempting to speculate that Drosophila has not only evolved tissue-specific $\mathrm{TAF}_{\mathrm{II}} \mathrm{s}$ but perhaps an entirely new functionally specialized TBP/TAF isocomplex that could be composed largely of testis-specific components (Fig. 5, bottom right).

\section{Mammalian TAF1 and TAF10 are required for cell cycle progression}

Biochemical fractionation of mammalian cell extracts revealed distinct TFIID populations that differ in their 
specific $\mathrm{TAF}_{\mathrm{II}}$ composition (Bell and Tora 1999). Interestingly, hsTAF10 (formerly human $\mathrm{TAF}_{\mathrm{II}} 30$ ) was found to be present in only $50 \%$ of all TFIID complexes (Jacq et al. 1994). Subsequent KO experiments in murine carcinoma cells revealed that cells lacking mouse TAF10 are blocked in the $\mathrm{G}_{1} / \mathrm{G}_{0}$ phase of the cell cycle and undergo apoptosis (Metzger et al. 1999). These findings suggested that TAF10 is required for the expression of a subset of genes important for cell cycle progression and viability in embryonal murine carcinoma cells; however, specific target genes of TAF10 remain elusive and it is unclear whether TAF10 truly serves as a tissue- or cell typespecific subunit of TFIID.

The findings regarding TAF10 are reminiscent of a temperature-sensitive mutation in TAF1 (formerly $\mathrm{TAF}_{\mathrm{II}} 250$ ) that also leads to cell cycle arrest and apoptosis (Talavera and Basilico 1977; Sekiguchi et al. 1995). The expression of cell cycle regulation genes such as cyclins A, D1, D3, and cdc2 are compromised when ts13 cells bearing a mutant TAF1 are grown at the restrictive temperature (Wang and Tjian 1994; Suzuki-Yagawa et al. 1997; Wang et al. 1997; O'Brien and Tjian 1998, 2000). Interestingly, the acetyl-transferase activity of TAF1 is also impaired at the restrictive temperature suggesting that this catalytic activity of TAF1 may be required for the proper expression of genes involved in the regulation of cell cycle and apoptosis (Dunphy et al. 2000).

Another potential example of TAF diversification involves alternatively spliced transcripts encoding three isoforms of hsTAF6 (formerly hTAF 70 or hTAF hII $_{\text {II }}$ ) designated hsTAF6a,b,c (Weinzierl et al. 1993). Human and Drosophila TAF6 directly interacts with the TFIID subunits TAF1 and TBP and recruits TAF9 into the TFIID complex (Weinzierl et al. 1993). Bell et al. (2001) identified a differentially spliced isoform of hsTAF6 (hsTAF6d) that is proteolytically cleaved upon apoptotic stimuli. Interestingly, overexpression of hsTAF6d, as well as hsTAF6a, induces cell death in HeLa cells and it appears that hsTAF6d is part of a TFIID-like complex lacking TAF6 and TAF9 (and other canonical TAFs) in which hsTAF6d displaces hsTAF9 (Bell et al. 2001). This is consistent with the previous finding that depletion of TAF9 in chicken DT40 cells causes apoptosis (Chen and Manley 2000). These findings raise the possibility that specific TAF isoforms may be generated by alternative splicing and post-translational modification in response to extracellular signaling events that could change the composition and presumably the activity of TFIID to regulate gene expression.

\section{Revisiting mechanisms of promoter selectivity}

In this review we have summarized evidence that metazoan organisms evolved functionally specialized transcription initiation complexes and promoter-selectivity modules that might serve as metazoan $\sigma$ factors responsible for coordinating the transcription of functionally related gene sets (Gross et al. 1998; Losick 1998). Two common themes have emerged from the studies described here. First, there are TFIID complexes containing cell type-specific isoforms of the canonical TAFs (i.e., the ovary-specific mTAF4b or the testis-specific dmTAF5 homolog cannonball) that function as cell typespecific counterparts of TFIID subunits (Fig. 5). Second, the TBP-related factors TRF1 and TRF2 exhibit an alternate core promoter specificity that is modulated by distinct sets of associated proteins (i.e., TRF1/BRF or TRF2/ DREF) that are structurally unrelated to the canonical TAF subunits of the prototypic core promoter recognition complexes (Fig. 2).

The discovery of TRF1 as the first cell type-specific homolog of a general transcription factor seemed like an oddity and was largely considered an exception to the rule. However, the discovery of a large and growing number of cell type-specific and tissue-specific isoforms of general transcription factors suggests that metazoan organisms exploit this variability to expand promoter selectivity and tissue-specific gene expression in a more general fashion than originally anticipated. Strikingly, both in flies and mammals, the majority of the tissuespecific isoforms of TAFs are involved in germ cell development. Likewise, at least mouse TRF2 also appears to be important for germ cell development. What is so special about germ cells? Why would germ cell development in particular require specialized transcription initiation complexes? A hallmark that distinguishes germ cells from other tissues is that they undergo meiosis and then develop into specialized haploid cells. Unlike most other cell types, germ cells form diploid zygotes that are by definition pluripotent. As a consequence, their cellular identity is not restricted to a given cell type dictated by a progenitor cell (e.g., a muscle cell after cell division will remain a muscle cell). Instead, germ cells are reprogrammed to differentiate into many cell types. Another potential unique aspect of germ cells is the stability of their genome and the importance of avoiding errors during replication and transcription. Perhaps specialized mechanisms to monitor genome stability and ensure transcriptional fidelity require a more elaborate and robust gene expression apparatus. Indeed, it has been observed that ovaries and testis express high levels of many different classes of transcription factors representing a considerably broader range than is typically expressed in differentiated somatic cells. It is thus conceivable that the high fidelity reprogramming that occurs in germ cells involves cell-specific, functionally specialized transcription initiation complexes that enable germ cells to accurately activate many different gene networks required for proper embryonic development.

The evolution of TBP-related factors also adds another layer of complexity to the regulatory mechanisms that govern gene expression in metazoans. For example, the modular composition of the Drosophila TRF2 complex combines promoter-selective components (DREF) with what appear to be potential chromatin remodeling factors (i.e., ISWI, NURF-55, NURF-38; Hochheimer et al. 2002; Fig. 4). The remarkable association between transcription factors and chromatin remodeling activities opens up the possibility for some novel mechanisms. For instance, DREF was recently reported to bind the scs' 
chromatin boundary element and evidence was presented that DREF competes with the boundary elementbinding factor BEAF for occupancy at overlapping sites in vivo (Hart et al. 1999). Intriguingly, several of the TRF2associated factors contain multiple Zinc finger motifs that resemble proteins implicated in silencing and Insulator function (Hochheimer et al. 2002). This leads us to hypothesize that the DREF-containing TRF2 complex may also participate in regulating Insulator activity.

Remarkably, BEAF and DREF can compete for occupancy at overlapping binding sites in vitro, and Drosophila salivary gland polytene chromosome staining revealed that BEAF and DREF bind in a mutually exclusive manner at select genomic sites (Hart et al. 1999). While DREF dissociates from the chromosomes in metaphase, BEAF remains associated throughout mitosis and might therefore serve as an epigenetic marker to re-establish chromatin boundaries after mitosis. These results suggest an antagonistic interplay between BEAF and DREF and are consistent with the idea that DREF may play some role in counteracting Insulator activity. It is thus likely that the recruitment of a chromatin-modifying activity residing in the DREF-containing TRF2 complex could create transcriptionally competent chromatin by counteracting the repressive effects of transcriptionally inactive heterochromatin.

With a wide range of functional approaches now available complemented by rigorous in vitro biochemical studies it should be possible to divide this complex transcription machinery into a manageable number of functional modules. A detailed analysis of individual components within these modules should enable us to untangle this network of multisubunit regulators and help dissect the mechanisms employed by metazoan organisms to govern the coordinate control of transcription during growth, differentiation, and development.

\section{Acknowledgments}

We thank Yvonne Berghöfer-Hochheimer, Rich Freiman, Ken Geles, Brad Glover, Yoh Isogai, Jim Kadonaga, Tim Kutzkey, Richard Losick, Susan Mango, and Stephen Smale for comments on the manuscript and helpful discussions.

\section{References}

Ainbinder, E., Revach, M., Wolstein, O., Moshonov, S., Diamant, N., and Dikstein, R. 2002. Mechanism of rapid transcriptional induction of tumor necrosis factor $\alpha$-responsive genes by NF-кB. Mol. Cell. Biol. 22: 6354-6362.

Albright, S.R. and Tjian, R. 2000. TAFs revisited: More data reveal new twists and confirm old ideas. Gene 242: 1-13.

Aoyagi, N. and Wassarman, D.A. 2000. Genes encoding Drosophila melanogaster RNA polymerase II general transcription factors: Diversity in TFIIA and TFIID components contributes to gene-specific transcriptional regulation. I. Cell Biol. 150: F45-F50.

Becker, P.B. and Horz, W. 2002. ATP-dependent nucleosome remodeling. Annu. Rev. Biochem. 71: 247-273.

Bell, B. and Tora, L. 1999. Regulation of gene expression by multiple forms of TFIID and other novel $\mathrm{TAF}_{\mathrm{II}^{-}}$-containing complexes. Exp. Cell Res. 246: 11-19.

Bell, B., Scheer, E., and Tora, L. 2001. Identification of hTAF 80 $\delta$ links apoptotic signaling pathways to transcription factor TFIID function. Mol. Cell 8: 591-600.

Berger, S.L. 2002. Histone modifications in transcriptional regulation. Curr. Opin. Genet. Dev. 12: 142-148.

Burley, S.K. and Roeder, R.G. 1996. Biochemistry and structural biology of transcription factor IID (TFIID). Annu. Rev. Biochem. 65: 769-799.

Butler, J.E. and Kadonaga, J.T. 2002. The RNA polymerase II core promoter: A key component in the regulation of gene expression. Genes \& Dev. 16: 2583-2592.

Chen, Z. and Manley, J.L. 2000. Robust mRNA transcription in chicken DT40 cells depleted of $\mathrm{TAF}_{\mathrm{II}} 31$ suggests both functional degeneracy and evolutionary divergence. Mol. Cell. Biol. 20: 5064-5076.

Comai, L., Tanese, N., and Tjian, R. 1992. The TATA-binding protein and associated factors are integral components of the RNA polymerase I transcription factor, SL1. Cell 68: 965976.

Crowley, T.E., Hoey, T., Liu, J.K., Jan, Y.N., Jan, L.Y., and Tjian, R. 1993. A new factor related to TATA-binding protein has highly restricted expression patterns in Drosophila. Nature 361: 557-561.

Dantonel, J.C., Wurtz, J.M., Poch, O., Moras, D., and Tora, L. 1999. The TBP-like factor: An alternative transcription factor in Metazoa? Trends Biochem. Sci. 24: 335-339.

Dantonel, J.C., Quintin, S., Lakatos, L., Labouesse, M., and Tora, L. 2000. TBP-like factor is required for embryonic RNA polymerase II transcription in C. elegans. Mol. Cell 6: 715722.

Dikstein, R., Ruppert, S., and Tjian, R. 1996a. TAF 250 is a bipartite protein kinase that phosphorylates the base transcription factor RAP74. Cell 84: 781-790.

Dikstein, R., Zhou, S., and Tjian, R. 1996b. Human TAF 105 is a cell type-specific TFIID subunit related to hTAF 1 II 13 . Cell 87: 137-146.

Dunphy, E.L., Johnson, T., Auerbach, S.S., and Wang, E.H. 2000. Requirement for $\mathrm{TAF}_{\mathrm{II}} 250$ acetyltransferase activity in cell cycle progression. Mol. Cell. Biol. 20: 1134-1139.

Dynlacht, B.D., Hoey, T., and Tjian, R. 1991. Isolation of coactivators associated with the TATA-binding protein that mediate transcriptional activation. Cell 66: 563-576.

Freiman, R.N., Albright, S.R., Zheng, S., Sha, W.C., Hammer, R.E., and Tjian, R. 2001. Requirement of tissue-selective TBP-associated factor TAF $_{\text {II }} 105$ in ovarian development. Science 293: 2084-2087.

Freiman, R.N., Albright, S.R., Chu, L.E., Zheng, S., Liang, H.E., Sha, W.C., and Tjian, R. 2002. Redundant role of tissue-selective $\mathrm{TAF}_{\mathrm{II}} 105$ in $\mathrm{B}$ lymphocytes. Mol. Cell. Biol. 22: 6564-6572.

Geiduschek, E.P. and Kassavetis, G.A. 1995. Comparing transcriptional initiation by RNA polymerases I and III. Curr. Opin. Cell Biol. 7: 344-351.

Geiman, T.M. and Robertson, K.D. 2002. Chromatin remodeling, histone modifications, and DNA methylation-how does it all fit together? J. Cell Biochem. 87: 117-125.

Goodrich, J.A. and Tjian, R. 1994. TBP-TAF complexes: Selectivity factors for eukaryotic transcription. Curr. Opin. Cell Biol. 6: 403-409.

Gross, C.A., Chan, C., Dombroski, A., Gruber, T., Sharp, M., Tupy, J., and Young, B. 1998. The functional and regulatory roles of $\sigma$ factors in transcription. Cold Spring Harb. Symp. Quant. Biol. 63: 141-155.

Hansen, S.K., Takada, S., Jacobson, R.H., Lis, J.T., and Tjian, R. 1997. Transcription properties of a cell type-specific TATA- 
binding protein, TRF. Cell 91: 71-83.

Hart, C.M., Cuvier, O., and Laemmli, U.K. 1999. Evidence for an antagonistic relationship between the boundary elementassociated factor BEAF and the transcription factor DREF. Chromosoma 108: 375-383.

Hernandez, N. 1993. TBP, a universal eukaryotic transcription factor? Genes \& Dev. 7: 1291-1308.

Hiller, M.A., Lin, T.Y., Wood, C., and Fuller, M.T. 2001. Developmental regulation of transcription by a tissue-specific TAF homolog. Genes \& Dev. 15: 1021-1030.

Hirose, F., Yamaguchi, M., Handa, H., Inomata, Y., and Matsukage, A. 1993. Novel 8-base pair sequence (Drosophila DNA replication-related element) and specific binding factor involved in the expression of Drosophila genes for DNA polymerase $\alpha$ and proliferating cell nuclear antigen. J. Biol. Chem. 268: 2092-2099.

Hobbs, N.K., Bondareva, A.A., Barnett, S., Capecchi, M.R., and Schmidt, E.E. 2002. Removing the vertebrate-specific TBP N terminus disrupts placental $\beta 2 \mathrm{~m}$-dependent interactions with the maternal immune system. Cell 110: 43-54.

Hochheimer, A., Zhou, S., Zheng, S., Holmes, M.C., and Tjian, R. 2002. TRF2 associates with DREF and directs promoterselective gene expression in Drosophila. Nature 420: 439445.

Hoey, T., Weinzierl, R.O., Gill, G., Chen, J.L., Dynlacht, B.D., and Tjian, R. 1993. Molecular cloning and functional analysis of Drosophila TAF110 reveal properties expected of coactivators. Cell 72: 247-260.

Holmes, M.C. and Tjian, R. 2000. Promoter-selective properties of the TBP-related factor TRF1. Science 288: 867-870.

Jacobson, R.H., Ladurner, A.G., King, D.S., and Tiian, R. 2000. Structure and function of a human $\mathrm{TAF}_{\mathrm{II}} 250$ double bromodomain module. Science 288: 1422-1425.

Jacq, X., Brou, C., Lutz, Y., Davidson, E., Chambon, P., and Tora, L. 1994. Human $\mathrm{TAF}_{\mathrm{II}} 30$ is present in a distinct TFIID complex and is required for transcriptional activation by the estrogen receptor. Cell 79: 107-117.

Jasper, H., Benes, V., Atzberger, A., Sauer, S., Ansorge, W., and Bohmann, D. 2002. A genomic switch at the transition from cell proliferation to terminal differentiation in the Drosophila eye. Dev. Cell 3: 511-521.

Kaltenbach, L., Horner, M.A., Rothman, J.H., and Mango, S.E. 2000. The TBP-like factor CeTLF is required to activate RNA polymerase II transcription during C. elegans embryogenesis. Mol. Cell 6: 705-713.

Kim, J.L., Nikolov, D.B., and Burley, S.K. 1993. Co-crystal structure of TBP recognizing the minor groove of a TATA element. Nature 365: 520-527.

Lemon, B. and Tjian, R. 2000. Orchestrated response: A symphony of transcription factors for gene control. Genes \& Dev. 14: 2551-2569.

Losick, R. 1998. Summary: Three decades after $\sigma$. Cold Spring Harb. Symp. Quant. Biol. 63: 653-666.

Maldonado, E. 1999. Transcriptional functions of a new mammalian TATA-binding protein-related factor. J. Biol. Chem. 274: $12963-12966$.

Malik, S. and Roeder, R.G. 2000. Transcriptional regulation through Mediator-like coactivators in yeast and metazoan cells. Trends Biochem. Sci. 25: 277-283.

Martianov, I., Fimia, G.M., Dierich, A., Parvinen, M., SassoneCorsi, P., and Davidson, I. 2001. Late arrest of spermiogenesis and germ cell apoptosis in mice lacking the TBP-like TLF/TRF2 gene. Mol. Cell 7: 509-515.

Metzger, D., Scheer, E., Soldatov, A., and Tora, L. 1999. Mammalian $\mathrm{TAF}_{\mathrm{II}} 30$ is required for cell cycle progression and specific cellular differentiation programmes. EMBO $J$. 18:
4823-4834

Mizzen, C.A., Yang, X.J., Kokubo, T., Brownell, J.E., Bannister, A.J., Owen-Hughes, T., Workman, J., Wang, L., Berger, S.L., Kouzarides, T., et al.. 1996. The $\mathrm{TAF}_{\mathrm{II}} 250$ subunit of TFIID has histone acetyltransferase activity. Cell 87: 1261-1270.

Moore, P.A., Ozer, J., Salunek, M., Jan, G., Zerby, D., Campbell, S., and Lieberman, P.M. 1999. A human TATA binding protein-related protein with altered DNA binding specificity inhibits transcription from multiple promoters and activators. Mol. Cell. Biol. 19: 7610-7620.

Muller, F., Lakatos, L., Dantonel, J., Strahle, U., and Tora, L. 2001. TBP is not universally required for zygotic RNA polymerase II transcription in zebrafish. Curr. Biol. 11: 282-287.

Naar, A.M., Lemon, B.D., and Tjian, R. 2001. Transcriptional coactivator complexes. Annu. Rev. Biochem. 70: 475-501.

Narlikar, G.J., Fan, H.Y., and Kingston, R.E. 2002. Cooperation between complexes that regulate chromatin structure and transcription. Cell 108: 475-487.

O'Brien, T. and Tjian, R. 1998. Functional analysis of the human $\mathrm{TAF}_{\mathrm{II}} 250 \mathrm{~N}$-terminal kinase domain. Mol. Cell 1: 905 911

- 2000. Different functional domains of $\mathrm{TAF}_{\mathrm{II}} 250$ modulate expression of distinct subsets of mammalian genes. Proc. Natl. Acad. Sci. 97: 2456-2461.

Ohbayashi, T., Kishimoto, T., Makino, Y., Shimada, M., Nakadai, T., Aoki, T., Kawata, T., Niwa, S., and Tamura, T. 1999a. Isolation of cDNA, chromosome mapping, and expression of the human TBP-like protein. Biochem. Biophys. Res. Commun. 255: 137-142.

Ohbayashi, T., Makino, Y., andTamura, T.A. 1999b. Identification of a mouse TBP-like protein (TLP) distantly related to the Drosophila TBP-related factor. Nucleic Acids Res. 27: 750-755.

Ohler, U., Liao, G.C., Niemann, H., and Rubin, G.M. 2002. Computational analysis of core promoters in the Drosophila genome. Genome Biol. 3: 1-12.

Orphanides, G. and Reinberg, D. 2002. A unified theory of gene expression. Cell 108: 439-451.

Ozer, J., Moore, P.A., and Lieberman, P.M. 2000. A testis-specific transcription factor IIA (TFIIA $\tau$ ) stimulates TATA-binding protein-DNA binding and transcription activation. $J$. Biol. Chem. 275: 122-128.

Pham, A.D. and Sauer, F. 2000. Ubiquitin-activating/conjugating activity of $\operatorname{TAF}_{\mathrm{II}} 250$, a mediator of activation of gene expression in Drosophila. Science 289: 2357-2360.

Rabenstein, M.D., Zhou, S., Lis, J.T., and Tjian, R. 1999. TATA box-binding protein (TBP)-related factor 2 (TRF2), a third member of the TBP family. Proc. Natl. Acad. Sci. 96: 47914796.

Roeder, R.G. 1996. The role of general initiation factors in transcription by RNA polymerase II. Trends Biochem. Sci. 21: 327-335.

Sekiguchi, T., Nakashima, T., Hayashida, T., Kuraoka, A., Hashimoto, S., Tsuchida, N., Shibata, Y., Hunter, T., and Nishimoto, T. 1995. Apoptosis is induced in BHK cells by the tsBN462/13 mutation in the CCG1/TAF 250 subunit of the TFIID basal transcription factor. Exp. Cell Res. 218: 490498.

Sharp, P.A. 1992. TATA-binding protein is a classless factor. Cell 68: 819-821.

Smale, S.T. 2001. Core promoters: Active contributors to combinatorial gene regulation. Genes \& Dev. 15: 2503-2508.

Strahl, B.D. and Allis, C.D. 2000. The language of covalent histone modifications. Nature 403: 41-45.

Suzuki-Yagawa, Y., Guermah, M., and Roeder, R.G. 1997. The ts 13 mutation in the $\mathrm{TAF}_{\mathrm{II}} 250$ subunit (CCG1) of TFIID di- 
rectly affects transcription of D-type cyclin genes in cells arrested in $\mathrm{G}_{1}$ at the nonpermissive temperature. Mol. Cell. Biol. 17: 3284-3294.

Takada, S., Lis, J.T., Zhou, S., and Tjian, R. 2000. A TRF1:BRF complex directs Drosophila RNA polymerase III transcription. Cell 101: 459-469.

Talavera, A. and Basilico, C. 1977. Temperature sensitive mutants of BHK cells affected in cell cycle progression. J. Cell Physiol. 92: 425-436.

Tanese, N., Saluja, D., Vassallo, M.F., Chen, J.L., and Admon, A. 1996. Molecular cloning and analysis of two subunits of the human TFIID complex: hTAF 130 and $\mathrm{hTAF}_{\mathrm{II}} 100$. Proc. Nat1. Acad. Sci. 93: 13611-13616.

Teichmann, M., Wang, Z., Martinez, E., Tjernberg, A., Zhang, D., Vollmer, F., Chait, B.F., and Roeder, R.G. 1999. Human TATA-binding protein-related factor-2 (hTRF2) stably associates with hTFIIA in HeLa cells. Proc. Natl. Acad. Sci. 96: $13720-13725$.

Upadhyaya, A.B., Lee, S.H., and DeJong, J. 1999. Identification of a general transcription factor TFIIA $\alpha / \beta$ homolog selectively expressed in testis. J. Biol. Chem. 274: 18040-18048.

Upadhyaya, A.B., Khan, M., Mou, T.C., Junker, M., Gray, D.M., and DeJong, J. 2002. The germ cell-specific transcription factor ALF. Structural properties and stabilization of the TATAbinding protein (TBP)-DNA complex. I. Biol. Chem. 277: 34208-34216.

Veenstra, G.J. and Wolffe, A.P. 2001. Gene-selective developmental roles of general transcription factors. Trends Biochem. Sci. 26: 665-671.

Veenstra, G.J., Weeks, D.L., and Wolffe, A.P. 2000. Distinct roles for TBP and TBP-like factor in early embryonic gene transcription in Xenopus. Science 290: 2312-2315.

Verrijzer, C.P. and Tjian, R. 1996. TAFs mediate transcriptional activation and promoter selectivity. Trends Biochem. Sci. 21: 338-342.

Wang, E.H. and Tjian, R. 1994. Promoter-selective transcriptional defect in cell cycle mutant ts 13 rescued by hTAF 250 . Science 263: 811-814.

Wang, E.H., Zou, S., and Tjian, R. 1997. TAF 250 -dependent transcription of cyclin A is directed by ATF activator proteins. Genes \& Dev. 11: 2658-2669.

Weinzierl, R.O., Ruppert, S., Dynlacht, B.D., Tanese, N., and Tjian, R. 1993. Cloning and expression of Drosophila $\mathrm{TAF}_{\mathrm{II}} 60$ and human $\mathrm{TAF}_{\mathrm{II}} 70$ reveal conserved interactions with other subunits of TFIID. EMBO J. 12: 5303-5309.

White-Cooper, H., Schafer, M.A., Alphey, L.S., and Fuller, M.T. 1998. Transcriptional and post-transcriptional control mechanisms coordinate the onset of spermatid differentiation with meiosis I in Drosophila. Development 125: 125134.

Wolstein, O., Silkov, A., Revach, M., and Dikstein, R. 2000. Specific interaction of $\mathrm{TAF}_{\mathrm{II}} 105$ with OCA-B is involved in activation of octamer-dependent transcription. J. Biol. Chem. 275: 16459-16465.

Yamit-Hezi, A. and Dikstein, R. 1998. TAF 105 mediates activation of anti-apoptotic genes by NF-кB. EMBO I. 17: 51615169.

Yamit-Hezi, A., Nir, S., Wolstein, O., and Dikstein, R. 2000. Interaction of $\mathrm{TAF}_{\mathrm{II}} 105$ with selected p65/RelA dimers is associated with activation of subset of NF-к B genes. J. Biol. Chem. 275: 18180-18187.

Zhang, D., Penttila, T.L., Morris, P.L., Teichmann, M., and Roeder, G. 2001. Spermiogenesis deficiency in mice lacking the Trf2 gene. Science 292: 1153-1155. 


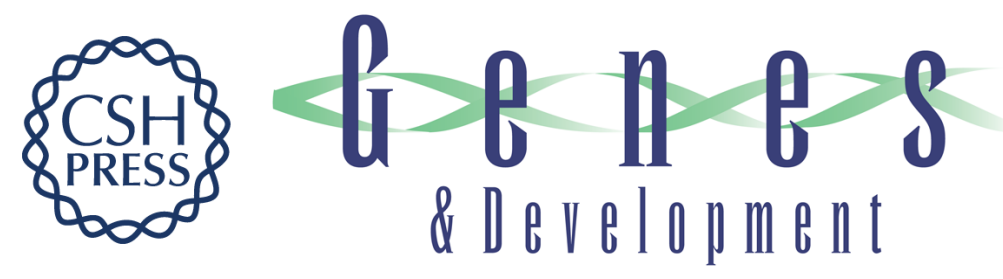

\section{Diversified transcription initiation complexes expand promoter selectivity and tissue-specific gene expression}

Andreas Hochheimer and Robert Tjian

Genes Dev. 2003, 17:

Access the most recent version at doi:10.1101/gad.1099903

References This article cites 79 articles, 33 of which can be accessed free at: http://genesdev.cshlp.org/content/17/11/1309.full.html\#ref-list-1

License

Email Alerting

Receive free email alerts when new articles cite this article - sign up in the box at the top Service right corner of the article or click here.

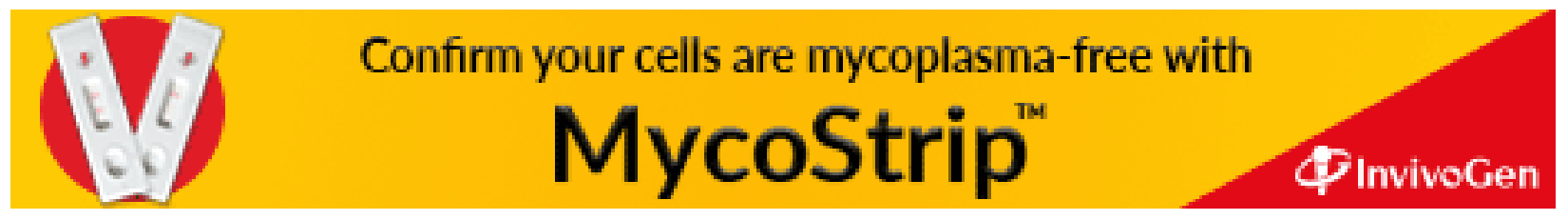

